\title{
Static and Dynamic Effects of Lateral Carrier Diffusion in Semiconductor Lasers
}

\author{
Jianzhong Li, Samson H. Cheung, and C. Z. Ning \\ the Center for Nanotechnology and NAS, \\ NASA Ames Research Center, M/S T27A-1, Moffett Field, CA 94035, U.S.A.
}

\begin{abstract}
Electron and hole diffusions in the plane of semiconductor quantum wells play an important part in the static and dynamic operations of semiconductor lasers. It is well known that the value of diffusion coefficients affects the threshold pumping current of a semiconductor laser. At the same time, the strength of carrier diffusion process is expected to affect the modulation bandwidth of an $\mathrm{AC}$-modulated laser. It is important not only to investigate the combined $\mathrm{DC}$ and $\mathrm{AC}$ effects due to carrier diffusion, but also to separate the $\mathrm{AC}$ effects from that of the combined effects in order to provide design insights for high speed modulation. In this presentation, we apply a hydrodynamic model developed by the present authors recently from the semiconductor Bloch equations. The model allows microscopic calculation of the lateral carrier diffusion coefficient, which is a nonlinear function of the carrier density and plasma temperature. We first studied combined $\mathrm{AC}$ and $\mathrm{DC}$ effects of lateral carrier diffusion by studying the bandwidth dependence on diffusion coefficient at a given DC current under small signal modulation. The results show an increase of modulation bandwidth with decrease in the diffusion coefficient. We simultaneously studied the effects of nonlinearity in the diffusion coefficient. To clearly identify how much of the bandwidth increase is a result of decrease in the threshold pumping current for smaller diffusion coefficient, thus an effective increase of DC pumping, we study the bandwidth dependence on diffusion coefficient at a given relative pumping. A detailed comparison of the two cases will be presented.
\end{abstract}

Keywords: semiconductor laser, carrier diffusion, nonlinearity, current modulation

\section{INTRODUCTION}

It is known that inhomogeneity in semiconductor lasers influence their operations. ${ }^{1-11}$ Such non-uniformity can result from both extrinsic and intrinsic sources. For example, interface roughness and disordered alloying profile cause additional scattering for carriers, and thus reduce their mobility and diffusivity. Additionally, current spreading or crowding due to inadequate design can lead to excessive thermal budget, increase in injection current threshold, and reduction in slope efficiency for the active device. In particular, non-uniform carrier distribution and the associated diffusion process in the lateral quantum well (QW) layer have been shown, both experimentally and numerically, to affect dynamic behaviors, ${ }^{2}$ modulation response, ${ }^{3}$ mode dynamics ${ }^{4}$ and selection, ${ }^{5}$ beam quality and optoelectric property ${ }^{6}$ threshold current, ${ }^{7}$ etc. Using a density-dependent diffusion coefficient, Sarzala and Nakwaski ${ }^{9}$ and the present authors ${ }^{11}$ concluded that an average constant value of the coefficient is sufficient for reliable simulation results, which will be shown in this work the need for quantitative modification.

Despite all the efforts, no correlation has been established for the effects of lateral diffusion on the DC operation and $\mathrm{AC}$-modulation performance. In other words, how much of the change in the AC-modulation performance because of lateral diffusion is derived from the corresponding change in the DC characteristics? The answer to this question deepens our understanding of the static and dynamic nature ${ }^{12}$ of the effects of lateral diffusion and improves our insights for device design. Statically, lateral diffusion increases the threshold current and reduces the slope efficiency of an active device under influence, which in turn alters the device's relative pumping level. According to the rate-equation description with a linearized gain model and no diffusion, ${ }^{13}$ the corresponding spectral response under AC-modulation scales with the relative injection level and thus changes in full correlation. So it becomes

Further author information:

J.Z.L.: E-mail: jianzhng@nas.nasa.gov

S.H.C.: E-mail: cheung@nas.nasa.gov

C.Z.N.: E-mail: cning@nas.nasa.gov 
an apparent question: Will this prediction change in a description with a fully nonlinear gain model in addition to inclusion of diffusive process? In this work, we present such a model, select the relatively simple single mode gain-guided VCSEL case, and show that within the limit of our study, it can be concluded that the effects of lateral diffusion on AC-modulation (termed AC effects later, in comparison with the DC effects) are purely of static nature, which indicates that the $\mathrm{AC}$ effects are a result of changes in the $\mathrm{DC}$ operation characteristics, i.e., the threshold injection current and the slope efficiency. Definitely, possible changes are rich and non-trivial for cases such as a laterally-coupled strip laser (refer to the special session for this specific case in this proceedings) or a VCSEL featuring transverse mode dynamics. Additionally it is unambiguously shown that the nonlinear diffusion coefficient can be substituted by an equivalent constant coefficient as far as both DC and AC-modulation performance are concerned. A quantitative criterion is established to locate the constant coefficient.

The paper is organized as follows. In the following section, theoretical aspect of our work will be summarized. The complete set of equations for our physical model is given together with expressions for the nonlinear density diffusion coefficient. Then in Section 3 , we first present the microscopically calculated diffusion coefficient as a function of plasma density and temperature, followed by numerical results and discussions for both DC operation and AC-modulation. We intend to identify the static and dynamic nature of the lateral carrier diffusion effects on AC-modulation by first applying a scaling scheme and then lineshape analysis for the spectral response under AC-modulation. Finally, the paper is concluded with a summary and some concluding remarks.

\section{PHYSICAL MODEL AND EQUATIONS}

The present work is based on a hydrodynamic model we developed recently ${ }^{10,14,15}$ to describe the intra-QW carrier dynamics of a semiconductor laser. Next, we summarize the relevant equations and briefly explain the physics involved. First of all, our model explicitly considers differentiation between carrier types, which then can be conveniently reduced to a single-component description-the ambipolar diffusion (AD) regime-under certain approximations. This is the regime considered in this work. Furthermore, we neglect plasma heating effects in this study in order to reduce the associated complications. Their effects are topics of future study.

In the $\mathrm{AD}$ regime, Poisson equation is eliminated due to charge neutrality and the whole set of equations for the description of the plasma density $N$, the complex transverse laser near field envelop $\mathcal{E}$, and the macroscopic polarization $P$, within the QW plane $\vec{r}$, is listed below:

$$
\begin{gathered}
\partial_{t} N+\partial_{\vec{r}} \cdot\left(-D_{N N} \partial_{\vec{r}} N\right)=-\gamma_{N} N+\frac{\eta J}{e}-\frac{L_{m} \Gamma}{4 \hbar} \Im\left(P^{*} \mathcal{E}\right), \\
\partial_{t} \mathcal{E}=\frac{i v_{g}}{2 K} \partial_{\vec{r}}^{2} \mathcal{E}+\frac{i v_{g} K \Gamma}{2 \epsilon_{0} \epsilon_{b}} P-\kappa \mathcal{E}, \\
\partial_{t} P_{1}=\left\{-\Gamma_{1}\left(N, T_{l}\right)+i\left[\omega_{c}-\omega_{1}\left(N, T_{l}\right)\right]\right\} P-i \epsilon_{0} \epsilon_{b} A_{1}\left(N, T_{l}\right) \mathcal{E}, \\
P=P_{0}+P_{1} ; \quad P_{0}=\epsilon_{0} \epsilon_{b} \chi_{0}\left(N, T_{l}\right) \mathcal{E},
\end{gathered}
$$

where $J$ is the injection current with a spatial pumping profile and quantum efficiency $\eta, L_{m}$ is the aggregate width of the active region, and $\Gamma$ is the optical mode confinement factor. Shorthand notation for the partial derivatives is used, such as $\partial_{t} N \equiv \partial N / \partial t$. Other notations in the above equations are standard, for example, $\omega_{c}$ is the central frequency and $v_{g}$ is the group velocity of light in the background material.

In the above set of equations, the macroscopic polarization and laser field equations are treated following Ref. 16. The polarization $P$ is decomposed into two parts, electronic and background, as expressed by Eq. 4, where the background contribution $P_{0}$ is given in a constitutive relation as well. The electronic part is governed dynamically by Eq. 3. The parameters- the effective background susceptibility $\chi_{0}$, the gain bandwidth $\Gamma_{1}$, the peak gain frequency $\omega_{1}$, and the Lorentzian oscillator strength $A_{1}$-are fitted to microscopically computed results as a function of carrier density $N$ and lattice temperature $T_{l}{ }^{16}$ The laser field is obtained after integrating over the assumed longitudinal mode profile.

Distinctively in our model, the ambipolar diffusion coefficient $D_{N N}$ is obtained microscopically by taking into account many-body effects within the Hartree-Fock approximation where carrier and polarization scatterings are treated under second-order Born approximation. The coefficient is found to be contributed by both free carrier and 
many-body parts. Specifically, it is dependent upon the scattering rates of electrons and holes with the longitudinal optical (LO) phonons in a semiconductor QW as listed below:

$$
D_{N N}=\left(\partial_{N} W+N \partial_{N} \delta \epsilon_{g}\right) /\left(m_{e} \gamma_{L O}^{e}+m_{h} \gamma_{L O}^{h}\right)
$$

where $W$ is the plasma thermal energy, $\delta \epsilon_{g}$ is the bandgap renormalization owing to the Coulomb interaction between electrons and holes, $m_{e, h}$ and $\gamma_{L O}^{e, h}$ are the masses and scattering rates of electrons and holes.

\section{SIMULATION RESULTS AND DISCUSSIONS}

Now we present and discuss our numerical results based on the above equations, which have taken into account the nonlinearity in both the material gain and the diffusion coefficient in the $\mathrm{AD}$ regime. The simulations are run for a single mode gain-guided MQW GaAs/InGaAs VCSEL operating at an $\omega_{c}$ of $980 \mathrm{~nm}$. The injection current profile is uniformly circular with a diameter of $7.5 \mu \mathrm{m}$ in the middle and smoothly reduced to zero at $8.25 \mu \mathrm{m}$ following a squared cosinoidal waist shape. The cavity length $L$ is $144 \mathrm{~nm}$ and $L_{m}$ is $4 \times 9 \mathrm{~nm}=36 \mathrm{~nm}$, which yields a $\Gamma$ of 0.25 . The other VCSEL parameters and simulation details can be found in Ref. 17. Except the carrier lifetime $\left(\tau_{n r}=1 / \gamma_{N}\right)$ of 0.25 nanosecond, all rates are computed microscopically and fitted as a function of plasma density $N$ and lattice temperature $T_{l}$ for our simulations. Furthermore, we use an injection current $J$ within $50 \%$ above threshold current. Numerical results in this work are for near field at the center of the device, which is representative for single mode operation. The lattice temperature $T_{l}$, as well as the plasma temperature $T_{p}$, is assumed to be a constant of $295 \mathrm{~K}$ throughout our simulations. Finally, we use a finite difference method to solve the time-dependent equations (1-4) in the $\mathrm{QW}$ plane.

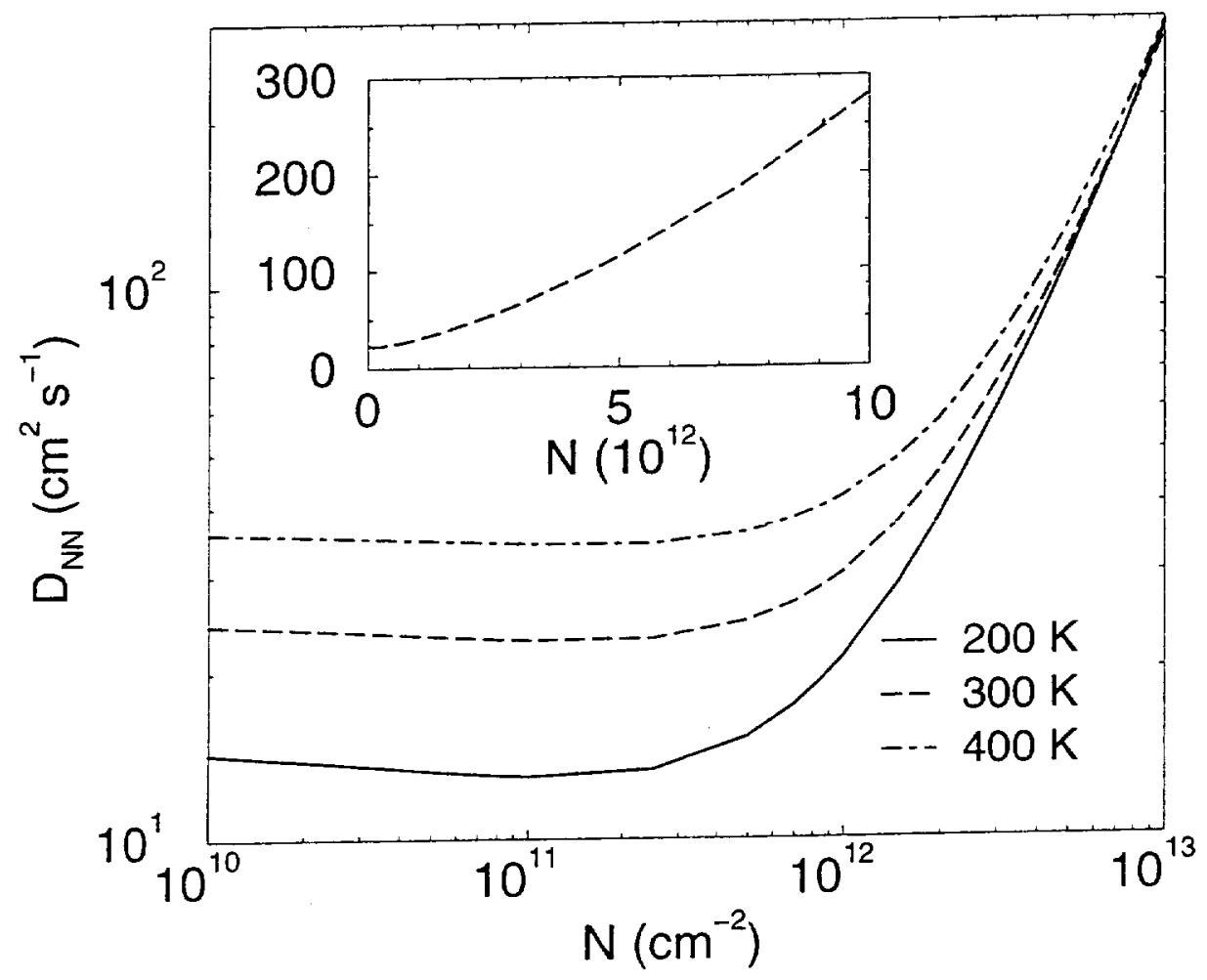

Figure 1. Ambipolar diffusion coefficients vs. plasma density for an 8 nanometer InGaAs quantum well.

First, we show the ambipolar diffusion coefficient for the InGaAs quantum well as a function of plasma density and temperature in Fig. 1. Here we only illustrate the nonlinearity in the diffusion coefficient and focus on its understanding from the physical point of view. Overall, the coefficient increases with plasma temperature, but in the intermediate density range many-body effects reduce it by as much as $20 \%$ in magnitude. ${ }^{18}$ At low density where 
classical statistics applies, both the plasma thermal energy term and the carrier-LO phonon scattering rates become density-independent and linearly proportional to the plasma temperature, whereas the bandgap renormalization term is negligible. By contrast, when the carriers become statistically degenerate at high density, the thermal energy term becomes the dominant contribution and increases with density linearly, whereas the scattering rates weakly decrease with density due to screening effects. As a result, the diffusion coefficient becomes a nonlinear function in the plasma density around the transparency point $\left(1.9 \times 10^{12} \mathrm{~cm}^{-2}\right.$ in this study). Later in this section, we will show that this nonlinear diffusion coefficient $D_{N N}$ can be substituted with an equivalent constant one as far as DC operations and AC-modulation performance are concerned.
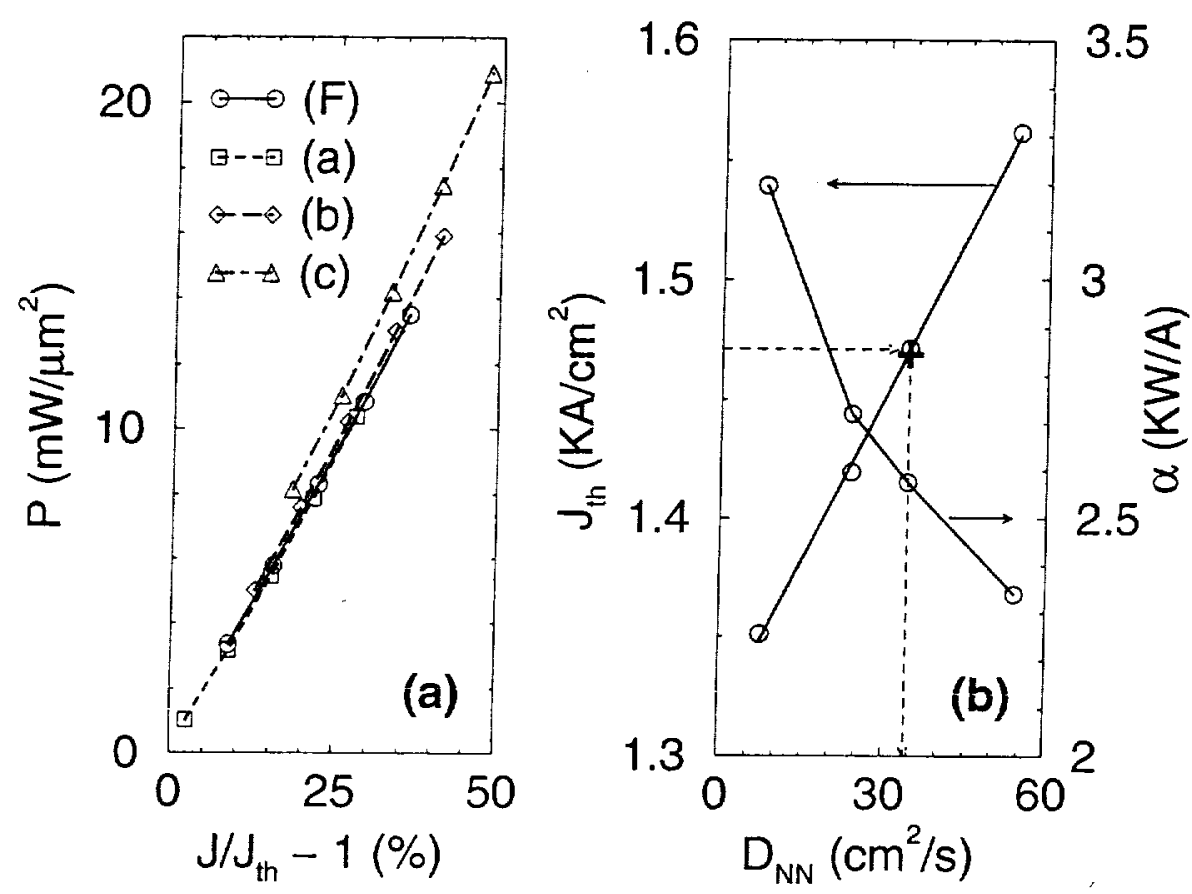

Figure 2. DC effects of lateral diffusion: (a) Near-field laser intensity vs. relative injection level $J / J_{t h}-1$ for four cases. Case (F): solid curve with circle symbols for nonlinear $D_{N N}$, and cases (a)-(c): constant $D_{N N} \mathrm{~s}$; (b) threshold current (circle symbols only) and slope efficiency (solid curve with circle symbols) vs. diffusion coefficient. The solid curve through the threshold current data points is a linear fit to the data. The equivalent constant coefficient data have been added (at $34.34 \mathrm{~cm}^{2} / \mathrm{s}$ ). Refer to Figure 5(a) and text for further explanation. Denoted with a plus symbol is the nonlinear diffusion result.

Next, we present the numerical DC operating characteristics of the VCSEL. The results are presented in a scaling manner where the relative injection level is adopted as the scaling parameter. As mentioned, the field intensity is for the center point of the device so that a constant conversion factor is required to obtain the far field intensity, and so does the slope efficiency. Nevertheless, this conversion does not affect our conclusions. Throughout this work, we choose four cases with representative diffusion coefficients to show the effects of lateral diffusion. Case $(F)$ corresponds to employment of the full nonlinear diffusion coefficient $D_{N N}(N)$ in simulation, case (a) features a constant coefficient of $54.115 \mathrm{~cm}^{2} / \mathrm{s}$ which corresponds to a plasma density of $2.5 \times 10^{12} \mathrm{~cm}^{-2}$ in relation with $D_{N N}(N)$, case (b) has $23.838 \mathrm{~cm}^{2} / \mathrm{s}$ which corresponds to $7 \times 10^{11} \mathrm{~cm}^{-2}$, and case (c) has $7.5 \mathrm{~cm}^{2} / \mathrm{s}$ which corresponds to $2.5 \times 10^{10} \mathrm{~cm}^{-2}$. We opt to work with these coefficients because (1) the nonlinear case (F) falls among them, for both $D C$ and $A C$ modulation characteristics; and (2) the range of values for the coefficients covers typical corresponding carrier density where appreciable change in the nonlinear coefficient is observed in Fig. 1. Therefore, the effects of lateral carrier density diffusion are demonstrated through comparison of the four cases. In Fig. 2, we plot the near field intensity as a function of the relative injection level $J / J_{t h}-1$ in Panel (a). As seen, both linear and nonlinear cases scale linearly with the relative injection level. This scaling behavior is referenced when we discuss the AC effects of lateral diffusion later. To better illustrate the effects, the threshold current (circle symbols only) and slope efficiency (solid 
curve with circle symbols) are plotted as a function of diffusion coefficient in Panel (b). Simultaneously shown in the plot are a linear fit to the threshold current and data points of the equivalent constant coefficient case (with a value of $34.34 \mathrm{~cm}^{2} / \mathrm{s}$ ) for the nonlinear case $(\mathrm{F})$. We shall discuss in detail the nonlinear case and its equivalent linear case later when the related result is presented in Fig. 5. Therefore, it is demonstrated that the induced increase in the threshold current by lateral carrier diffusion is linearly proportional to the diffusion coefficient. Correspondingly, a decrease in the slope efficiency results.
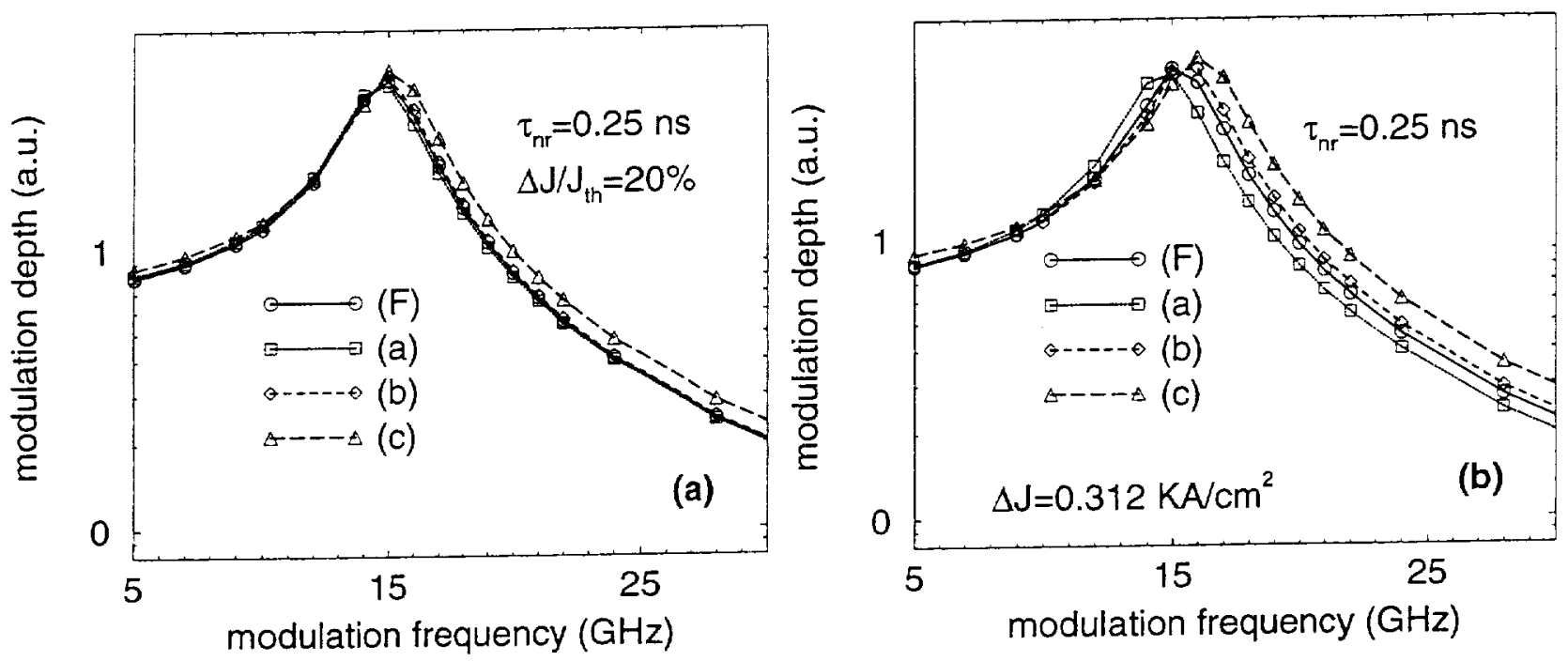

Figure 3. (a) AC-modulation spectral response at constant relative injection level of $20 \%$ for the same diffusion cases as in Figure 2; and (b) AC-modulation spectral response at constant difference injection level of $\Delta J=0.312 \mathrm{KA} / \mathrm{cm}^{2}$ for comparison.

As first seen from the DC effects of lateral diffusion presented above, the relative injection level seems to be a good controlling parameter for identifying the static and dynamic nature of the $\mathrm{AC}$ effects of lateral diffusion. Shown in Panel (a) of Fig: 3 are numerical results for the spectral response of the VCSEL under AC-modulation at the same relative injection level of $20 \%$ for the four diffusion cases: For comparison, in Panel (b) we plot the results at the same difference injection level of $\Delta J \equiv J-J_{t h}=0.312 \mathrm{KA} / \mathrm{cm}^{2}$. It is obvious that to some extent the results comparatively exhibit the expected scaling behavior for the spectral response when the $\mathrm{AC}$ effects of lateral diffusion is purely of static nature. However, due to the associated change in the slope efficiency with the threshold current, the evidence is not overwhelmingly convergent upon a clear identification. Therefore, we take these results as a strong hint for the purely static nature of the $\mathrm{AC}$ effects of lateral diffusion and move on to performing a lineshape analysis on the spectral response of the VCSEL.

Despite the fact that there is no known analytical guidance for lineshape analysis on the spectral response of a VCSEL under AC-modulation with diffusion process included, the suggestion comes from two sides. First, in reality, it is a common practice to model the spectral response using the classical linear rate-equation approach. ${ }^{13}$ Doing so reveals that the diffusive process affects the device physics of the VCSEL in such a manner that both the scaling behavior and spectral response are somewhat intact in functional form. Furthermore, the scaling behavior presented above provides additional support for a lineshape analysis since different responses tend to collapse together. The essence of a lineshape analysis lies in its ability to identify resonance position and dephasing mechanisms. In this work, we do not attempt to evaluate the latter. Instead, the analysis is used to find the former together with the peak amplitude and the results are checked to see whether they scale with the relative injection level. We argue that their scaling behavior would correlate with the DC effects of lateral diffusion and thus provide a strong but indirect evidence for identification of the static nature of the AC effects of lateral diffusion.

Now, we are ready to present such evidence. In Panel (a) of Fig. 4 we show spectral response results for the device at different injection levels but at a constant diffusion coefficient of $23.838 \mathrm{~cm}^{2} / \mathrm{s}$. One fit curve (dot-dashed curve) to the case with the largest injection level (dashed curve) is shown. The other fits similarly show agreement near resonance. The results tell a few things: First and foremost, the functional form of the lineshape is indeed intact 

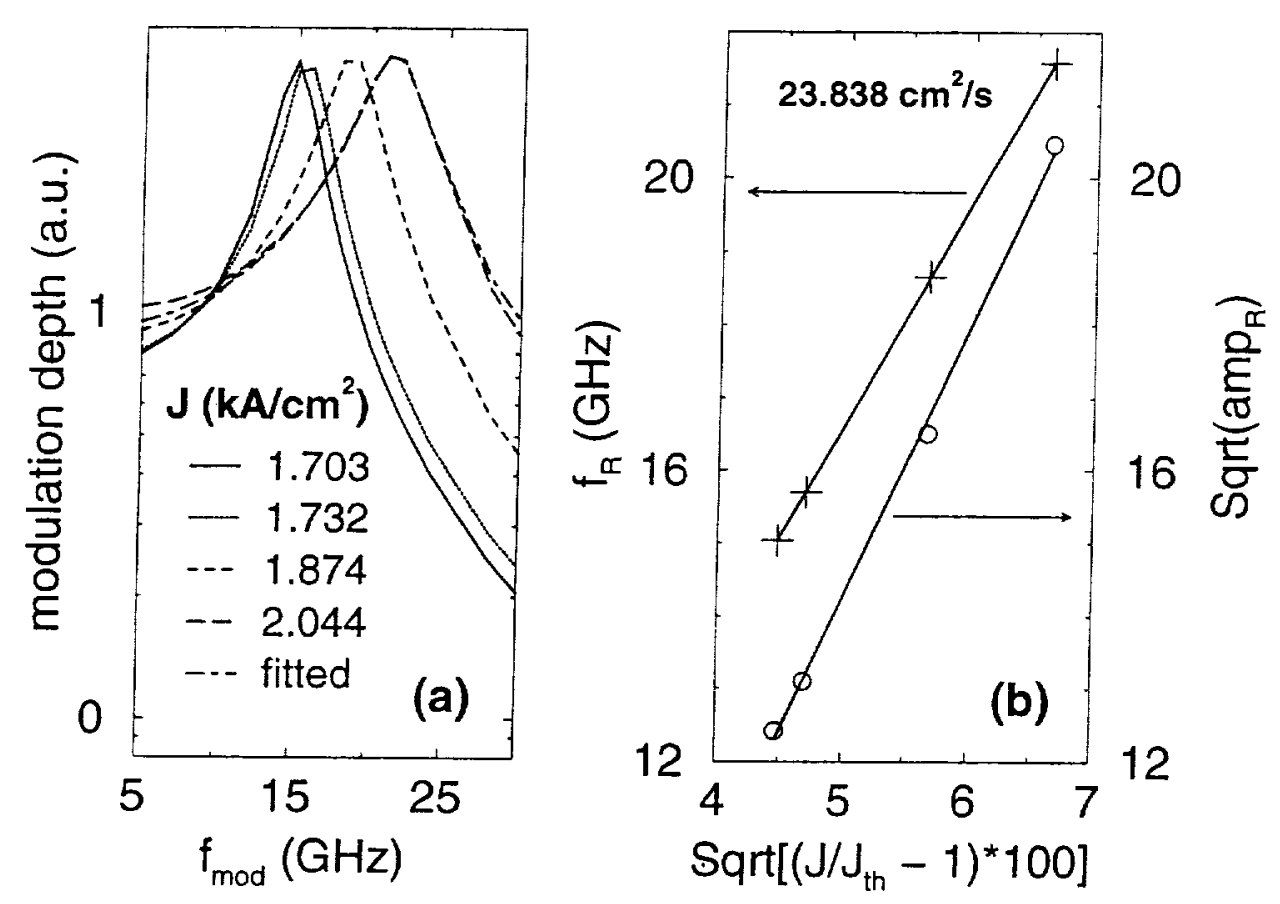

Figure 4. (a) AC-modulation spectral response at different injection levels and under a constant diffusion coefficient of $23.838 \mathrm{~cm}^{2} / \mathrm{s}$. The dot-dashed curve is a lineshape fit to the long dashed curve, as explained in the text; and (b) Resonance frequency (plus symbols only) and resonance amplitude (circle symbols only) vs. relative injection level. The solid curves are linear fit to the data points, respectively.

and given by $1 / \omega^{2}-\omega_{R}^{2}+i \omega \gamma \cdot{ }^{19}$ Second, diffusive process does cause growing deviations from this standard form at both low and high frequency sides. The nature of these deviations can be either static or dynamic or both, which is a subject of future study. Last, we point out that without an adequate assessment of the effects of diffusion, analysis performed based on the classical linear rate-equation model is expected to bear systematic discrepancy. Next, we show the resonance position and peak amplitude obtained from lineshape fitting as a function of the square root of the relative injection level in Panel (b). Note that in the plot the solid curves are linear fit to the data points. As seen, the resonance frequency scales with $\sqrt{J / J_{t h}-1}$ and the peak amplitude linearly depends on the relative injection level. The same scaling behavior is observed for the other diffusion cases, which are not shown. The scaling behavior of the resonance frequency is expected for a coupled two-component system where one component experiences gain from excess above threshold of the other component. Therefore, results shown here present the evidence we need to reach our conclusion that the $A C$ effects of lateral diffusion is purely of static nature, which is comprehensible from the physical point of view that diffusive process occurs at a slower timescale than the about $10 \mathrm{GHz}$ modulation rate.

It is interesting to take a closer examination of the classical linear rate-equation model and come to a more penetrating understanding of the effects of lateral diffusion. Basically the model ${ }^{13}$ features a linear gain approximation and no diffusion. For better discussion, we list the spectral response solution of the model below:

$$
p(\omega)=\frac{-\left(\frac{\delta J}{e}\right) A \Gamma \mathcal{P}_{0}}{\omega^{2}-i \omega\left(\gamma_{N}+A \mathcal{P}_{0}\right)-\kappa A \mathcal{P}_{0}}
$$

where $\delta J$ is the amplitude of AC-modulation current density, $A$ is the linear differential gain, and $\mathcal{P}_{0}$ is the $\mathrm{DC}$ laser field intensity. The resonance frequency is given from the above equation as $\omega_{R} \equiv \sqrt{\kappa A \mathcal{P}_{0}}=\sqrt{\gamma_{N}\left(\kappa+A \Gamma N_{t r}\right)} \times$ $\sqrt{J / J_{t h}-1}$ with $N_{t r}$ being the transparency density of the VCSEL. There are a few ways that lateral diffusion alters this description. As revealed in Panel (a) of Fig. 4, deviations are present at both low and high frequency sides. Furthermore, as demonstrated, the scaling behaviors of the resonance frequency and peak amplitude are still valid, but their scaling prefactors are diffusion-dependent, which shall be commented upon in the next concluding 
section. Also, analysis based on the classical model surely introduces systematic errors without taking into account the effects of lateral diffusion.
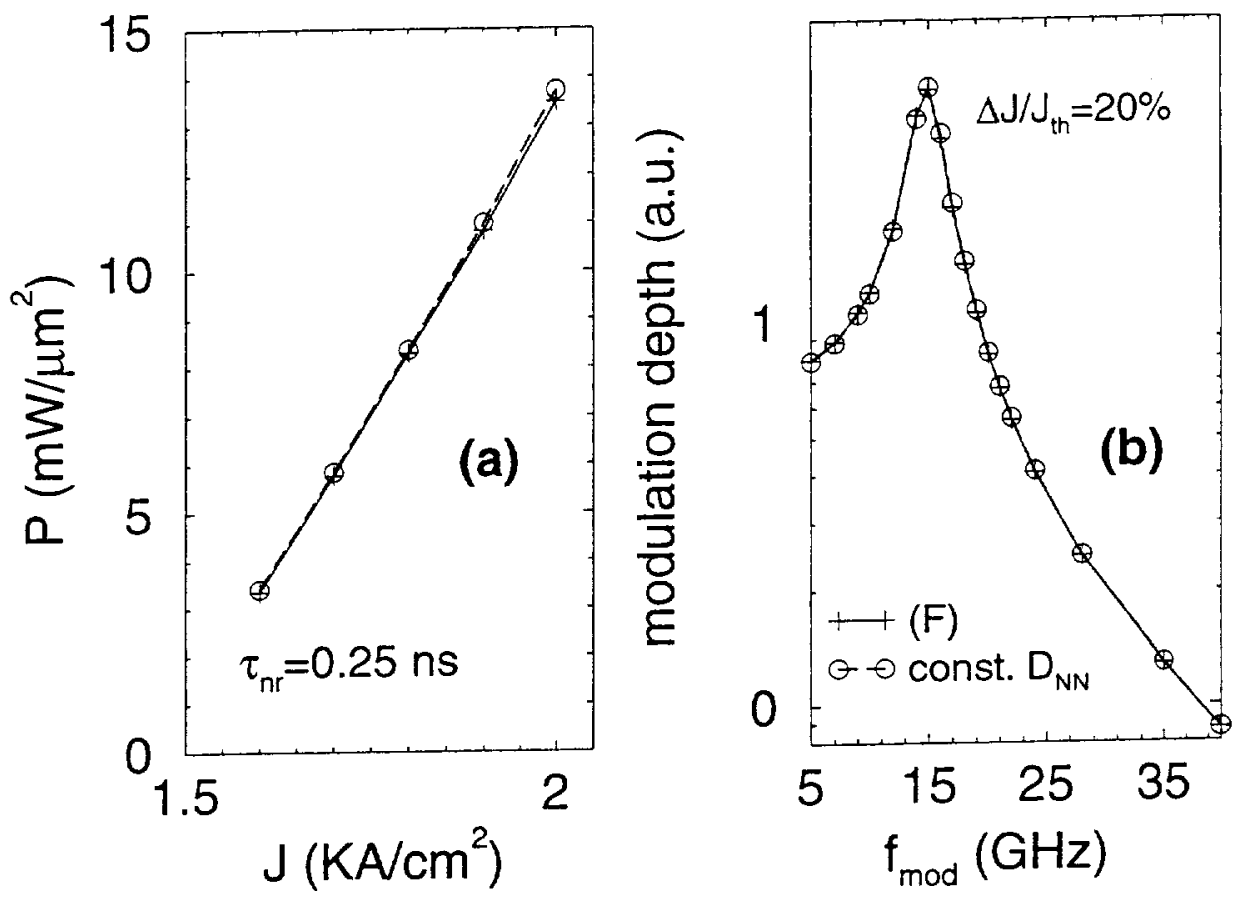

Figure 5. Comparison of (a) near-field laser intensity vs. injection level and (b) AC-modulation spectral response at the same relative injection level of $20 \%$ for the nonlinear diffusion coefficient case [ $(F)$ : solid curve with plus symbols] and its equivalent constant diffusion coefficient case [(const. $\left.D_{N N}\right)$ : long dashed curve with circle symbols].

Last, we show that an equivalent constant diffusion coefficient exists to reproduce the nonlinear diffusion characteristics by presenting a comparison of the DC and AC-modulation performance between the nonlinear diffusion case (F) with such a constant coefficient case. The coefficient for the latter case is chosen as follows: We first obtain the L-I curve for case (F) and find the corresponding threshold injection current. Then using the linear fit to the constant diffusion coefficient cases, as shown in Panel (b) in Fig. 2, we find the corresponding constant diffusion coefficient for the threshold current in case (F). This process is indicated in the figure by dashed lines with arrows pointing out the procedural flow. The simulation results are shown comparatively in Fig. 5 . Needless to say, the equivalence is established. There are a few points worth mentioning: First, we have given quantitative evidence for the equivalence and an unambiguous criterion to locate the value of the equivalent constant coefficient. Second, this criterion is quantitatively different from previous studies ${ }^{9,11}$ which pointed to 'an average constant value of the diffusion coefficient'. In this work, the average value, if given at half the pinned plasma density $\left(N_{\text {pin }}=\right.$ $1.893 \times 10^{12} \mathrm{~cm}^{-2}$ ) in the middle of the VCSEL, is $29.22 \mathrm{~cm}^{2} / \mathrm{s}$, which is about $15 \%$ below the equivalent coefficient of $34.34 \mathrm{~cm}^{2} / \mathrm{s}$. Certainly, ather averaging procedures could be introduced to reconcile with this offset, but our criterion is physically meaningful and straightforward. However, the reality is that the linear fit to the constant diffusion coefficient cases is nonexistent. Therefore, it brings out the last and most important point we would like to make, i.e., the nonlinearity in the diffusion coefficient is important in a self-consistent manner. Seemingly the equivalence of the nonlinear diffusion coefficient to a constant one should deny the necessity of a full nonlinear description of the diffusive process. We emphasize that this conclusion is restricted to the studied range and can not extrapolate without further investigation. Ramifications could result in other complex scenarios. Even in the restricted range, this equivalence has been established numerically through comparison. Therefore, it is impossible to identify the equivalent coefficient without first characterizing the nonlinear case. 


\section{SUMMARY AND CONCLUDING REMARKS}

In summary, we have presented the microscopically derived density diffusion coefficient as a function of plasma density in the $A D$ regime. The effects of lateral diffusion on $\mathrm{DC}$ operation and AC-modulation characteristics are studied through comparison of the nonlinear diffusion coefficient case with three constant coefficient cases and its equivalent constant coefficient case. Specifically, a scaling scheme and lineshape analysis are deployed in identification of the static and dynamic nature of the spectral response of the VCSEL under AC-modulation. The last numerical result presented is a direct comparison of the $\mathrm{DC}$ and $\mathrm{AC}$-modulation performance between the nonlinear diffusion case and its equivalent constant diffusion coefficient case.

Before closure of this paper, a few remarks are given. First, the nonlinearity in the diffusion coefficient does alter performance of the device. Second, despite the fact that we have shown that such a nonlinearity can be modeled with an equivalent constant coefficient, the answer to how to find the equivalent value is still pending. We hint here on how to proceed. As shown, the increase in the threshold current is proportional to the coefficient. Given that the proportionality coefficient is known, empirically or analytically, the equivalent constant coefficient is thus determined. By analyzing the AC-modulation response, if some analytical guidance were supplied, in principle we then should be able to obtain such critical device parameters as the differential gain, carrier lifetime, and photon lifetime. So we see a critical need for development of such an analytical understanding of the device with the effects of lateral diffusion included.

In conclusion, we have shown that within the studied range, i.e., $50 \%$ within injection current threshold for a single mode gain-guided VCSEL, not only the nonlinear diffusion coefficient can be replaced with an equivalent constant, but also a numerical scheme is provided to locate the constant. The numerical criterion established is that both coefficients yield the same threshold current. For the DC effects of lateral carrier density diffusion, it is found that the increase in threshold current depends linearly on the coefficient, whereas the slope efficiency decreases with diffusion coefficient. For the AC effects of lateral carrier density diffusion, it is found that, the spectral response follows the lineshape function of $1 / \omega^{2}-\omega_{R}^{2}+i \omega \gamma$ near resonance and deviates at low and high frequency sides: Both the resonance frequency $\omega_{R}$ and resonance amplitude scale with the relative injection level $J / J_{t h}-1$. Furthermore, using the scaling scheme and lineshape analysis, it is concluded that the AC effects of lateral carrier density diffusion is purely of static nature and are reflections of the corresponding change in the threshold current and slope efficiency. Finally, we emphasize the importance of taking into account nonlinearity in the diffusion coefficient at a self-consistent level.

\section{REFERENCES}

1. I. Vurgaftman and J. R. Meyer, "Effects of Bandgap, Lifetime, and Other Nonuniformities on Diode Laser Thresholds and Slope Efficiencies," IEEE J. Select. Topics Quantum Electron. 3, pp. 475-484, 1997.

2. N. Chinone, K. Aiki, M. Nakamura, and R. Ito, "Effects of lateral mode and carrier density profile on dynamic behaviors of semiconductor lasers," IEEE J. Quantum Electron. QE-14, pp. 625-631, 1978.

3. D. Wilt, K. Y. Lau, and A. Yariv, "The effect of lateral carrier diffusion on the modulation response of a semiconductor laser," J. Appl. Phys. 52, pp. 4970-4974, 1981.

4. P. A. Khandokhin, I. V. Koryukin, Y. I. Khanin, and P. Mandel, "Influence of Carrier Diffusion on the Dynamics of a Two-Mode Laser," IEEE J. Quantum Electron. 31, pp. 647-652, 1995.

5. N. K. Dutta, "Analysis of current spreading, carrier diffusion, and transverse mode guiding in surface emitting lasers," J. Appl. Phys. 68, pp. 1961-1963, 1990.

6. J.-W. Lai and C.-F. Lin, "Carrier Diffusion Effect in Tapered Semiconductor-Laser Amplifier," IEEE J. Quantum Electron. 34, pp. 1247-1256, 1998.

7. J. H. Shin, H. E. Shin, and Y. H. Lee, "Effect of carrier diffusion on oxidized vertical-cavity surface-emitting lasers determined from lateral spontaneous emission," Appl. Phys. Lett. 70, pp. 2652-2654, 1997.

8. R. S. Tucker and D. J. Pope, "Circuit Modeling of the Effect of Diffusion on Damping in a Narrow-Stripe Semiconductor Laser," IEEE J. Quantum Electron. QE-19, pp. 1179-1183, 1983.

9. R. P. Sarzala and W. Nakwaski, "Carrier diffusion inside active regions of gain-guided vertical-cavity surfaceemitting lasers," IEE Proc.-Optoelectron. 144, pp. 421-425, 1997.

10. J. Li, S. H. Cheung, and C. Z. Ning, "Theory and Simulation of Self- and Mutual-Diffusions of Carrier Density and Temperature in Semiconductor Lasers," in Physics and Simulation of Optoelectronic Devices IX, Y. Arakawa, P. Blood, and M. Osinski, eds., SPIE Proc. 4283, pp. 267-274, 2001. 
11. J. Li, S. H. Cheung, and C. Z. Ning, "Nonlinearity Effects of Lateral Density Diffusion Coefficient on Gainguided VCSEL Performance," in Optoelectronics, Materials, and Devices for Communications, T. P. Lee and Q. Wang, eds., SPIE Proc. 4580, pp. 52-59, 2001.

12. By dynamic nature we basically mean kinetics-related influence of the diffusive process on the AC-modulation response of a semiconductor laser, so its effects ought to be injection level-dependent and expected to deviate from the scaling behavior which we shall discuss later.

13. A. Yariv, Optical Electronics, Holt, Rinehart and Winston, New York, 1985.

14. J. Li and C. Z. Ning, "A Hydrodynamic Theory for Spatially Inhomogeneous Semiconductor Lasers: I. Microscopic Approach." submitted to Phys. Rev. A, 2002.

15. J. Li and C. Z. Ning, "A Hydrodynamic Theory for Spatially Inhomogeneous Semiconductor Lasers: II. Numerical Results." submitted to Phys. Rev. A, 2002.

16. C. Z. Ning, R. A. Indik, and J. V. Moloney, "Effective Bloch-equations for semiconductor lasers and amplifiers," IEEE J. Quantum Electron. 33, pp. 1543-1550, 1997.

17. C. Z. Ning and P. M. Goorjian, "Microscopic modeling and simulation of transverse-mode dynamics of verticalcavity-surface emitting lasers," J. Opt. Soc. Am. B 16, pp. 2072-2082, 1999.

18. C. Z."Ning and J. Li, "Many-body effects in a laterally inhomogeneous semiconductor quantum well." submitted to Phys. Rev. B, 2002.

19. The lineshape is determined by various interactions, such as that between laser field and plasma density, which gives the resonance behavior, and those incoherent ones between the coupled field-plasma system and the ambient, which produce the linewidth. In this work, vertical carrier transport and capture is not considered, so their corresponding contributions to the spectral response is missing, which is conventionally modeled with a multiplicative factor $1 / 1+i \omega \tau_{t c}$ where $\tau_{t c}$ is a lumped time constant accounting for the corresponding relaxational mechanisms. 
